\title{
UNA NUEVA LOCALIDAD DE PINUS DISCOLOR BAILEY \& HAWKSWORTH EN EL CENTRO DE MEXICO'
}

\author{
Fernando Zavala Chavez \\ Universidad Autónoma Chapingo \\ Apartado Postal 084 \\ Chapingo, 56230 Estado de México, México \\ Jose Luis Campos Diaz \\ Universidad Autónoma Chapingo \\ Departamento de Preparatoria Agrícola \\ Chapingo, 56230 Estado de México, México
}

\section{RESUMEN}

Se registra una nueva localidad para Pinus discolor Bailey \& Hawksworth en el centro de México, en la porción centro-oriental del estado de Querétaro. El límite sur de distribución conocida de la citada especie se extendía hasta cerca de los $22^{\circ} 02^{\prime}$ de latitud norte en el suroeste del estado de San Luis Potosí. Con esta información el límite meridional alcanza ahora aproximadamente los $20^{\circ} 50^{\prime} 45^{\prime \prime}$ de latitud norte en México.

\section{ABSTRACT}

The presence of Pinus discolor Bailey \& Hawksworth is reported from a new locality in Mexico: in the east-central part of the state of Querétaro. The southern limit of the known distribution of that species reached latitude $22^{\circ} 02^{\prime} \mathrm{N}$ in the southwestern part of the state of San Luis Potosi; with this report this limit is now extended down to latitude $20^{\circ} 50^{\prime} 45^{\prime \prime}$ in Mexico.

El área conocida de distribución de Pinus discolor Bailey \& Hawksworth se localiza a lo largo de la Sierra Madre Occidental, desde Arizona y Nuevo México en Estados Unidos de América, hasta el suroeste de San Luis Potosí, ya en el Altiplano de México. Se encuentra aproximadamente entre las coordenadas geográficas $31^{\circ} 45^{\prime}$ y $22^{\circ} 02^{\prime}$ de latitud norte y $101^{\circ} 45^{\prime}$ y $111^{\circ} 40^{\prime}$ longitud oeste (Bailey y Hawksworth, 1979, 1988; Bailey et al., 1982).

El informe proporcionado por el Ing. Antonio Plancarte Barrera, del Centro de Genética Forestal, A. C., sobre la venta de semilla con características semejantes a las

1 Trabajo realizado con apoyo de la Universidad Autónoma Chapingo. 
de Pinus discolor, en un poblado del estado de Querétaro, motivó la realización de exploraciones en las zonas de piñonar de la región centro-oriental de la entidad. La especie de pino piñonero, conocida localmente como "piñón gomoso", fue encontrada finalmente en dicha zona en octubre de 1991, fecha en la cual se realizaron las colectas del material botánico correspondiente por parte de los autores del presente estudio. Esto, en primera instancia, mostró que dicha especie se extiende más al sur de su distribución hasta entonces conocida.

El sitio donde se descubrió $P$. discolor se localiza en la Barranca de La Vaina, al sur del poblado de Los Juárez, aproximadamente a $8 \mathrm{~km}$ al norte de la población de Sombrerete perteneciente a la Delegación de Vizarrón, Municipio de Cadereyta, Querétaro. Se encuentra aproximadamente entre las coordenadas geográficas $20^{\circ} 50^{\prime} 45^{\prime \prime}$ y $20^{\circ} 51^{\prime}$ $30^{\prime \prime}$ latitud norte, y $99^{\circ} 38^{\prime} 30^{\prime \prime}$ y $99^{\circ} 40^{\prime}$ longitud oeste (Fig. 1). Las altitudes varían de 2300 a 2700 m (Anónimo, 1973). La vegetación es de piñonar de $P$. cembroides en las mayores altitudes y de $P$. discolor en las menores, con Nolina sp. ("soyate"), Rhus spp., Quercus pringlei y Q. repanda ("encino chico"), Juniperus flaccida y J. deppeana ("cedro"). Se trata de una ladera con exposición NNW a NNE, con pendientes pronunciadas hasta de $45^{\circ}-50^{\circ}$ y expuesta a fuerte erosión pluvial. El sustrato geológico es de roca caliza (Anónimo, 1974a); el terreno superficial es gravoso, con fragmentos menores de $7.5 \mathrm{~cm}$; el suelo es de tipo rendzina con textura gruesa a medianz en los primeros 30 $\mathrm{cm}$ de profundidad (Anónimo, 1974b).

En este piñonar existe una zona angosta donde se mezclan las poblaciones de Pinus cembroides y $P$. discolor, en la cual se detectaron individuos morfológicamente intermedios entre ambas especies. Esto se ratificó con los rasgos de las semillas; sin embargo, Bailey y Hawksworth (1979) y Bailey et al. (1982) mencionan la falta de evidencia de hibridación natural entre las dos especies.

La población de $P$. discolor de la Barranca de la Vaina consiste de pequeños árboles con alturas que van de 2 a 4(5) $\mathrm{m}$. Muchos se ramifican desde la base, pero otros presentan un tallo principal bien definido. El estudio de 55 individuos en edad reproductiva mostró la existencia de dioecia, encontrándose una proporción de sexos de 1.1 : 0.9 para femeninos y masculinos, respectivamente. Esto difiere de lo señalado por Bailey y Hawksworth (1979), quienes consignan que la dioecia en $P$. discolor es menos evidente hacia el límite meridional de su distribución. Sin embargo, coincide con lo indicado por Avila (1985), quien observó una proporción de sexos cercana a $1: 1$ en el suroeste del estado de San Luis Potosí.

Por otra parte, se colectaron muestras de 11 individuos de $P$. discolor, de las cuales se analizaron 50 fascículos; $98 \%$ de éstos presentaron 3 acículas y sólo $2 \%$ mostraron 4. La longitud promedio de las acículas fue mayor en los individuos femeninos que en los masculinos, alcanzando $4.3( \pm 0.7)$ y $3.9( \pm 0.5) \mathrm{cm}$, respectivamente. La edad de las hojas varió de 3 a 7 años. Estas características coinciden con las descritas para $P$. johannis (Robert, 1978) y P. discolor (Bailey y Hawksworth, 1979), especies supuestamente emparentadas de manera estrecha. Según Zavarin (1988), aún existe controversia en la delimitación taxonómica de ambas especies y las únicas caracteristicas que las separan son la distribución geográfica y algunos compuestos químicos diferentes que poseen. Sin embargo, la dioecia es un carácter importante de $P$. discolor (Bailey y Hawksworth, 1979), misma que no parece mostrar $P$. johannis (Robert, 1978). Las diferencias en la longitud promedio de acículas entre sexos y los datos sobre la edad de las acículas señalados anteriormente coinciden con lo indicado por Zavala et al. (1989), quienes encontraron 


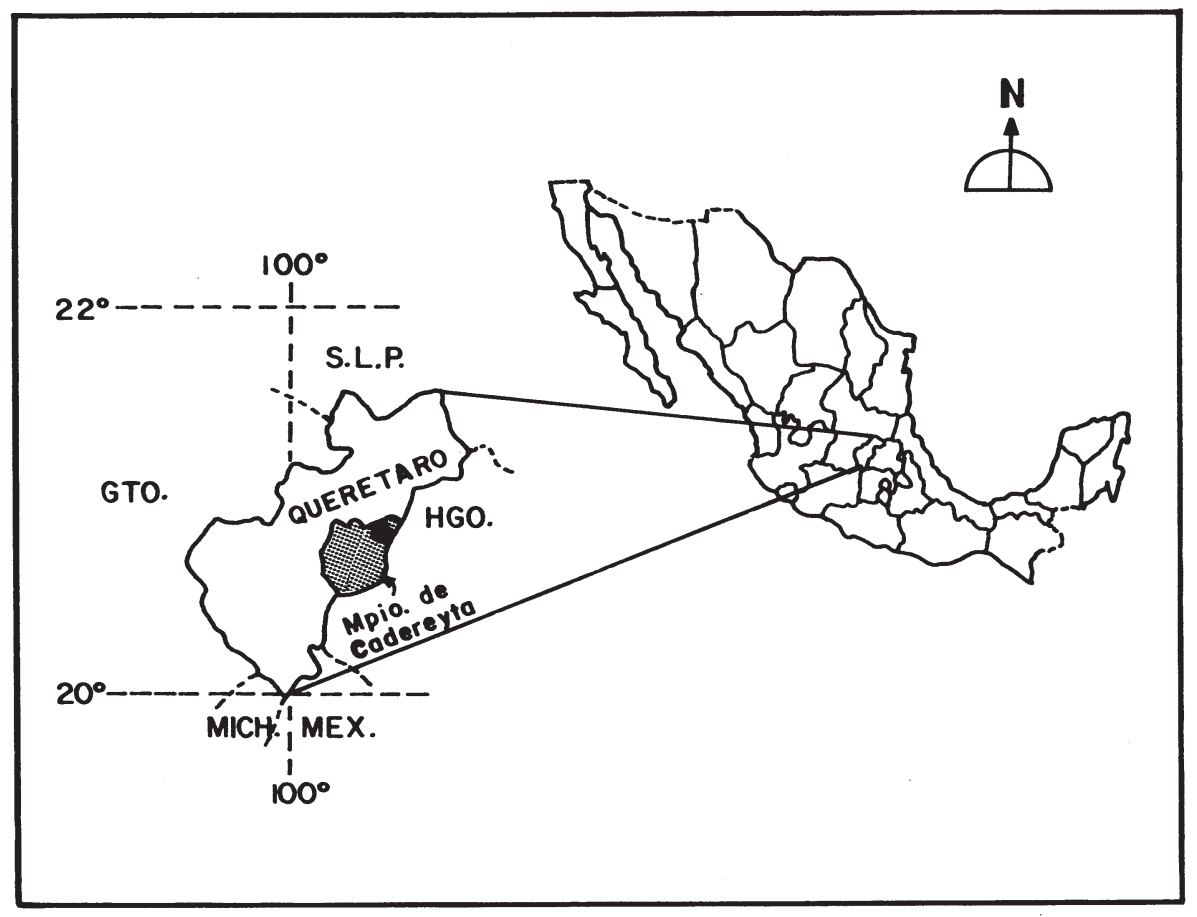

Fig. 1. Localización geográfica del área de estudio (pequeño círculo negro).

valores semejantes para $P$. discolor del poblado La Amapola, al suroeste del estado de San Luis Potosí.

El piñonar de la zona estudiada es objeto de recolección, principalmente cuando se espera que habrá alta producción de semilla. El nombre común de "piñón gomoso" para $P$. discolor se debe a la cantidad de resina que secretan sus conos femeninos maduros. Esta característica de la especie, además de lo insípido de sus semillas y lo duro de su testa, hacen que tenga poca demanda y por tal razón se colecta escasamente, aunque suele cosecharse para autoconsumo.

El presente informe adiciona una fracción más a las cuatro consignadas antes (Bailey y Hawksworth, 1988) y que forman un área discontínua en dirección de noroeste a sureste con relación al territorio mexicano. De esta manera, la distribución conocida de $P$. discolor queda ahora conformada por 5 porciones como sigue: a) al suroeste de Nuevo México y sureste de Arizona, E. U. A.; b) al sur de Arizona y suroeste de Nuevo México y noreste de Sonora y noroeste de Chihuahua, México; c) al sur de Chihuahua y noroeste de Durango; $c h)$ al suroeste de San Luis Potosi; y d) en el centro-oriente de Querétaro. Esta última constituye probablemente el límite meridional de distribución de $P$. discolor (Fig. 2), a los $20^{\circ} 51^{\prime} 30^{\prime \prime}$ de latitud norte, unos $190 \mathrm{~km}$ al sureste del manchón existente en el municipio de San Luis Potosí, S.L.P. 


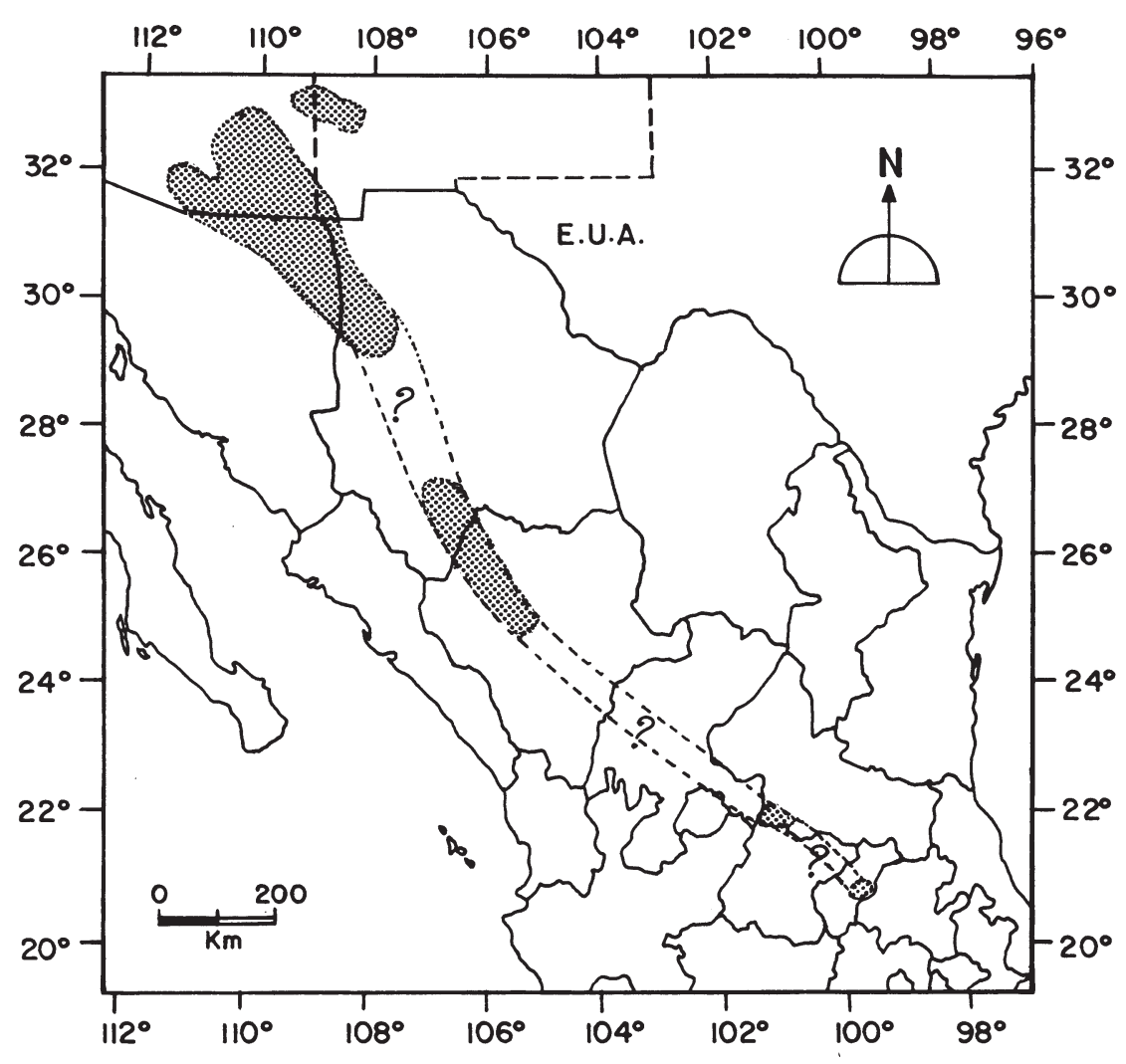

Fig. 2. Representación esquemática de la distribución geográfica conocida de Pinus discolor. (Modificado de Bailey y Hawksworth, 1988).

\section{AGRADECIMIENTOS}

Los autores agradecen al Ing. Antonio Plancarte Barrera, del Centro de Genética, A. C., por haber proporcionado la primera información sobre la posible presencia de $P$. discolor en Querétaro. Asímismo, se agradece la colaboración del C. Manuel Romero, de la Comunidad de Sombrerete, como guía en el recorrido por el área de interés. A la Universidad Autónoma de Chapingo por el apoyo para la realización de esta investigación. Al Dr. Jerzy Rzedowski Rotter, del Instituto de Ecología, A. C., por la revisión y atinadas sugerencias al manuscrito, y a los revisores anónimos de Acta Botánica Mexicana por sus observaciones. 


\section{LITERATURA CITADA}

Anónimo. 1972. Carta topográfica F-14-C-57, San Pablo Tolimán, Querétaro. Escala 1: 50 000. Comisión de Estudios del Territorio Nacional. México, D. F.

Anónimo. 1973. Carta topográfica F-14-C-57, San Joaquín, Querétaro-Hidalgo. Escala 1: 50 000. Comisión de Estudios del Territorio Nacional. México, D. F.

Anónimo. 1974 a. Carta geológica F-14-C-58, San Joaquín, Querétaro-Hidalgo. Escala 1: 50 000. Comisión de Estudios del Territorio Nacional. México, D. F.

Anónimo. 1974 b. Carta edafológica F-14-C-58, San Joaquín, Querétaro-Hidalgo. Escala 1: 50000. Comisión de Estudios del Territorio Nacional. México, D. F.

Avila, J. A. 1985. Caracterización de los piñoneros ( $P$. cembroides Zucc. y $P$. discolor Bailey y Hawks.) de las Serranías Meridionales del estado de San Luis Potosí, México. Tesis Profesional. Escuela de Biología. Universidad Michoacana de San Nicolás de Hidalgo. Morelia, Michoacán. 101 pp.

Bailey, D. K. y F. G. Hawksworth. 1979. Pinyons of the Chihuahuan Desert region. Phytologia 44 (3): $129-$ 133.

Bailey, D. K. y F. G. Hawksworth. 1988. Phytogeography and taxonomy of the pinyon pines Pinus subsection Cembroides. In: Passini, M. F., D. Cibrián T. y T. Eguiluz P. (comps.). Memorias del II Simposio Nacional sobre Pinos Piñoneros. Centre d'Etudes Mexicaines et Centramericaines Universidad Autónoma Chapingo - Centro de Genética Forestal, A. C. Chapingo, México. pp. 4164.

Bailey, D. K., K. Snajberk y E. Zavarin. 1982. On the question of natural hybridization between Pinus discolor and Pinus cembroides. Biochemical Systematics and Ecology 10 (2): 111-119.

Robert, M. F. 1978. Un nouveau pin pignon mexicain: Pinus johannis M.-F. Robert. Adansonia, ser. 2, 18 (3): $129-133$.

Zavala, F., J. A. Avila y E. García. 1989. Variación anual en la longitud de acículas y su relación con los factores ambientales en piñoneros del Altiplano Potosino. In: Flores F., J. D., J. Flores L., E. García M. y R. H. Lira S. (comps.). Memorias del III Simposio Nacional sobre Pinos Piñoneros. Universidad Autónoma Agraria Antonio Narro - Instituto Nacional de Investigaciones Forestales, Agrícolas y Pecuarias. Saltillo, Coahuila. pp. 72-80.

Zavarin, E. 1988. Taxonomy of pinyon pines. In: Passini, M. F., D. Cibrián T. y T. Eguiluz P. (comps.). Memorias del II Simposio Nacional sobre Pinos Piñoneros. México, D. F. Centre d'Etudes Mexicaines et Centramericaines - Universidad Autónoma Chapingo - Centro de Genética Forestal, A. C. Chapingo, México. pp. 29-40. 